\title{
Interplay between FGF21 and insulin action in the liver regulates metabolism
}

\author{
Brice Emanuelli, ${ }^{1}$ Sara G. Vienberg, ${ }^{1}$ Graham Smyth, ${ }^{1}$ Christine Cheng, ${ }^{2}$ \\ Kristin I. Stanford, ${ }^{1}$ Manimozhiyan Arumugam, ${ }^{3,4}$ Mervyn D. Michael, ${ }^{2}$ \\ Andrew C. Adams, ${ }^{2}$ Alexei Kharitonenkov, ${ }^{2}$ and C. Ronald Kahn'1
}

\author{
${ }^{1}$ Joslin Diabetes Center, Boston, Massachusetts, USA. ²illy Diabetes Research, Indianapolis, Indiana, USA. \\ ${ }^{3}$ Novo Nordisk Foundation Center for Basic Metabolic Research, Copenhagen University, Copenhagen, Denmark. ${ }^{4}$ BGI-Shenzhen, Shenzhen, China.
}

\begin{abstract}
The hormone FGF21 regulates carbohydrate and lipid homeostasis as well as body weight, and increasing FGF21 improves metabolic abnormalities associated with obesity and diabetes. FGF21 is thought to act on its target tissues, including liver and adipose tissue, to improve insulin sensitivity and reduce adiposity. Here, we used mice with selective hepatic inactivation of the IR (LIRKO) to determine whether insulin sensitization in liver mediates FGF21 metabolic actions. Remarkably, hyperglycemia was completely normalized following FGF21 treatment in LIRKO mice, even though FGF21 did not reduce gluconeogenesis in these animals. Improvements in blood sugar were due in part to increased glucose uptake in brown fat, browning of white fat, and overall increased energy expenditure. These effects were preserved even after removal of the main interscapular brown fat pad. In contrast to its retained effects on reducing glucose levels, the effects of FGF21 on reducing circulating cholesterol and hepatic triglycerides and regulating the expression of key genes involved in cholesterol and lipid metabolism in liver were disrupted in LIRKO mice. Thus, FGF21 corrects hyperglycemia in diabetic mice independently of insulin action in the liver by increasing energy metabolism via activation of brown fat and browning of white fat, but intact liver insulin action is required for FGF21 to control hepatic lipid metabolism.
\end{abstract}

\section{Introduction}

Obesity-associated metabolic complications, including metabolic syndrome and type 2 diabetes, are growing health problems worldwide $(1,2)$. Obesity results from an imbalance between energy intake and energy expenditure, which leads to increased adipose tissue mass and ectopic fat accumulation. This in turn triggers various defects in many organs including insulin resistance in fat, skeletal muscle and liver, hepatosteatosis, and pancreatic lipotoxicity, thus leading to type 2 diabetes and other related abnormalities such as accelerated atherosclerosis (3). Insulin resistance has been shown to be a crucial player in these processes (4), and therapeutic management of the metabolic syndrome or type 2 diabetes often involves pharmacologic treatment to increase insulin content or improve insulin sensitivity (5).

FGF21 is a novel hormone that has profound effects on metabolic parameters such as glucose and lipid homeostasis, in addition to promoting rapid body weight loss, and represents a promising potential therapy for the treatment of type 2 diabetes (6). Furthermore, transgenic mice overexpressing FGF21 are protected against diet-induced obesity (7), while mice in which FGF21 is ablated exhibit increased weight gain and impaired glucose tolerance when placed on a high-fat diet (HFD) (8). FGF21 appears to be primarily produced by the liver, where its expression is partially under the control of the transcription factor PPAR $\alpha$ (9-13). FGF21 expression is also induced by endogenous PPAR $\alpha$ ligands such as fatty acids, which increase after prolonged fasting (14), by a ketogenic diet in mice (9), or by fibrates, which are artificial PPAR $\alpha$ ligands $(11,15)$. In turn,

Conflict of interest: Christine Cheng, Mervyn D. Michael, Andrew C. Adams, and Alexei Kharitonenkov are employees of Eli Lilly and Company.

Citation for this article: J Clin Invest. 2014;124(2):515-527. doi:10.1172/JCI67353.
FGF21 affects whole-body substrate use by inhibiting lipolysis and lipid accumulation (16) in the white fat and triggering a ketogenic program in the liver (10). Hence, FGF21 appears to be an important factor in the overall regulation of metabolic processes, especially in the adaptation to fasting. A remarkable feature of this metabolic regulator is that exogenous administration of FGF21 to obese rodent models such as diet-induced obese (DIO) mice, $\mathrm{db} / \mathrm{db}$ or ob/ob mice, or diabetic monkeys, drastically and rapidly improves metabolic abnormalities, thus raising considerable therapeutic interest $(7,17)$. Indeed, FGF21 normalizes glycemia and insulin levels in insulin-resistant states, ameliorates dyslipidemia and reduces hepatosteatosis, corrects high cholesterol levels, decreases body weight, and restores insulin sensitivity in obese animals. However, the molecular mechanisms underlying FGF21 action are still poorly understood. FGF21-induced changes in total insulin sensitivity might mediate FGF21 metabolic effects. Also, it remains uncertain what tissues contribute to FGF21 efficacy. Two very recent studies indicated that FGF21 action in adipose tissue is critically required to mediate the majority of the metabolic beneficial effects of FGF21 $(18,19)$. Nevertheless, while adipose tissue is an important target for the metabolic effects of FGF21, both $\beta$ Klotho and FGF receptors, which are required for FGF21 action in vivo (20-23), are also present in the liver. Furthermore, previous studies have shown that effects such as FFA regulation remain in animals in which adipose FGFR1 is ablated (18).

The liver plays an essential role in the control of glucose and lipid homeostasis, and insulin is a central regulator of multiple hepatic metabolic functions. By modifying the expression and enzymatic activity of key players, insulin promotes glycogen synthesis, lipogenesis and lipoprotein synthesis, and inhibits gluconeogenesis, glycogenolysis, and VLDL secretion (24). Insulin action 

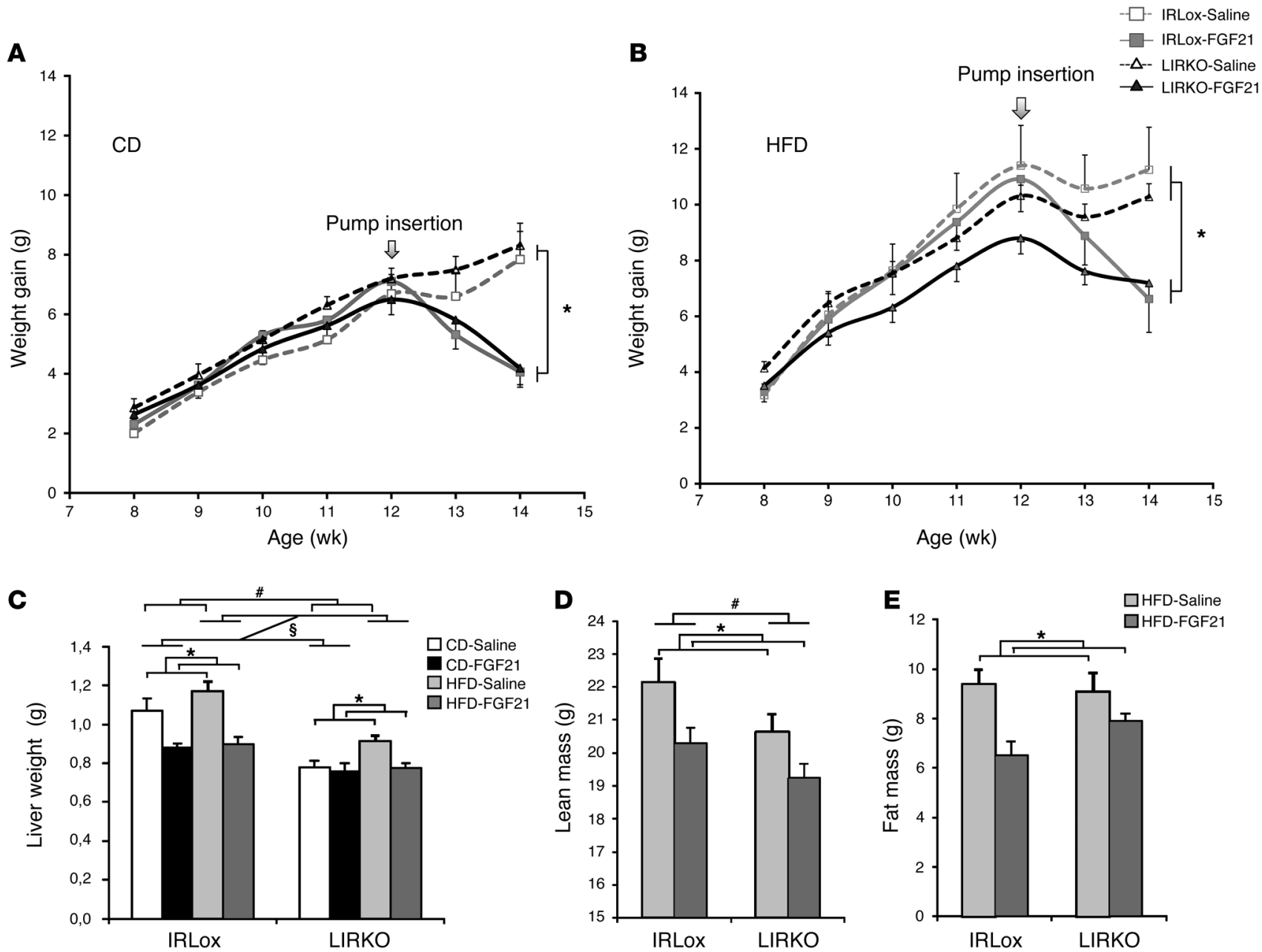

Figure 1

Effects of FGF21 on body and tissue weights. Control and LIRKO mice were fed either a CD or an HFD for 7 weeks and were treated with saline or FGF21 (1 mg/kg/day) delivered s.c. by osmotic pump during the last 2 weeks of the diet. Body weight was measured once a week, and tissue weights were determined on day 14 after insertion of the pump. (A and B) Body weight gain of animals on a CD (A) or an HFD (B). Gray lines with squares represent control mice, black lines with triangles represent LIRKO mice, dashed lines represent saline-treated mice, and solid lines represent FGF21-treated mice. (C) Liver weight. White bars represent saline-treated mice on a CD, black bars represent FGF21-treated mice on a $C D$, light gray bars represent saline-treated mice on an HFD, and dark gray bars represent FGF21-treated mice on an HFD. (D and E) Lean mass (D) and fat mass (E) from DXA analysis. Light gray bars represent saline-treated mice on an HFD, and dark gray bars represent FGF21-treated mice on an HFD. Data represent the means \pm SEM. $P$ values were calculated using 2 - or 3-way ANOVA. ${ }^{P}<0.05$ between genotypes; $\$ P<0.05$ between diets; ${ }^{*} P<0.05$ with FGF21 treatment. $n=5-12$ animals per group.

in the liver also plays a pivotal role in the control of cholesterol homeostasis (25). Therefore, hepatic insulin resistance contributes substantially to glucose intolerance and dyslipidemia.

Several lines of evidence suggest that FGF21 regulates hyperglycemia, at least in part, via the sensitization of insulin action, an effect we hypothesized is mediated by the liver. In obese animals, FGF21 lowers both glucose and insulin levels, suggesting an improvement in insulin sensitivity $(17,26)$. Hyperinsulinemic euglycemic clamp studies in ob/ob mice indicate that the effect of FGF21 on lowering blood glucose levels is associated with decreased hepatic glucose output, whereas no difference in glucose uptake in skeletal muscle and adipose tissue was observed (27), indicating an essential role for direct FGF21 action in the liver. Increased IR expression in liver following FGF21 treatment may partially account for this effect (26). FGF21 also inhibits hepatic lipid synthesis and controls gene expression involved in the regulation of both lipogenesis and lipid oxidation to reduce hepatosteatosis in obese animals (26). It remains unclear, however, whether the improvement in these metabolic parameters and the regulation of gene expression are directly caused by FGF21 action in the liver or are the result of changes in insulin sensitivity, circulating insulin levels, or reduced obesity.

In the present study, we defined the role of liver insulin sensitivity to FGF21 action by using mice with selective hepatic inactivation of the IR (LIRKO mice). These mice lack IRs in the liver, rendering them unable to regulate hepatic gluconeogenesis, and subsequently develop hyperglycemia (24) and hypercholesterolemia when on an HFD (25). Following the induction of obesity 
by HFD feeding to trigger obesity-related metabolic abnormalities, we compared the effects of FGF21 chronic infusion on the regulation of body weight and metabolic parameters, such as glucose and lipid metabolism, in both control and LIRKO mice. If the primary action of FGF21 is to reduce obesity and consequently restore insulin sensitivity in the liver, then the beneficial metabolic outcomes reported in control mice should be abolished in LIRKO mice. We first observed that FGF21 was equally efficacious in reducing body weight in both control and LIRKO mice. Strikingly, our results indicate that FGF21 is able to reverse diabetes independently of insulin sensitization in the liver. This effect was not due to the diminution of hepatic glucose output, as FGF21 had no effect on this parameter, but rather to increased glucose metabolism in brown adipose tissue (BAT), browning of white adipose tissue (WAT), and drastically increased energy expenditure. On the other hand, the regulation of circulating cholesterol and liver triglycerides by FGF21 was compromised in LIRKO mice and accompanied by the regulation of expression in the liver of key genes for carbohydrate and lipid metabolism. This suggests that the effects of FGF21 on lipid metabolism specifically require crosstalk with insulin action in the liver. Altogether, our data show that insulin sensitization in the liver is not required for FGF21 to correct hyperglycemia in diabetic mice, whereas intact liver insulin action is necessary to mediate the effects of FGF21 on lipid metabolism. Instead, we suggest that FGF21 acts via activation of BAT and browning of WAT, leading to increased energy metabolism and consequent shifts toward increased substrate use.

\section{Results}

FGF21 sensitivity in LIRKO animals. We first analyzed whether FGF21 sensitivity was affected by hepatic insulin resistance. Circulating FGF21 levels were similar in control and LIRKO mice, and FGF21 expression levels in liver and plasma were similarly increased in both fasting LIRKO mice and controls (Supplemental Figure 1, A and B; supplemental material available online with this article; doi:10.1172/JCI67353DS1), indicating that physiological regulation of FGF21 was intact in the LIRKO mice. Expression levels of FGF receptors $(F g f r)$ 1, 2, 3, and 4, as well as the coreceptor $\beta$ Klotho $(K l b)$, which constitute the FGF21 receptor machinery, were similar in control and LIRKO mice in all the putative FGF21 target tissues examined (perigonadal fat, s.c. fat, BAT, liver, skeletal muscle, pancreas, and hypothalamus) (Supplemental Figure 1C). Furthermore, acute i.p. injection of a maximal dose $(1 \mathrm{mg} / \mathrm{kg})$ of FGF21 resulted in a similar upregulation of Egr1 (as assessed by TaqMan analysis) in control and LIRKO animals in liver, pancreas, s.c., perigonadal and brown adipose tissues, and was absent in skeletal muscle and hypothalamus in these animals (Supplemental Figure 1D). Thus, control and LIRKO mice have similar FGF21 sensitivity, indicating that previously described doses of FGF21 should be adequate for our use in LIRKO mice.

Effectiveness of FGF21 in LIRKO animals. One well-characterized FGF21 action is its ability to lower body weight, and this has been proposed to be partially responsible for the amelioration of the metabolic profile of obese and diabetic mice $(17,26)$. To determine whether FGF21 could improve obesity and diabetes in animals deprived of insulin action in the liver, control and LIRKO mice were fed either a chow diet (CD) or an HFD for 7 weeks, then treated with either saline or FGF21 $(1 \mathrm{mg} / \mathrm{kg} /$ day $)$ by s.c. infusion during the last 2 weeks of the diet. During the pretreatment period, all mice gained weight, and this was enhanced by approxi- mately $25 \%$ in the control mice on an HFD and by approximately $10 \%$ in LIRKO mice on an HFD (Figure 1, A and B). Administration of FGF 21 induced a $7 \%-10 \%$ body weight loss in all groups of mice, i.e., those on a CD or an HFD and in those with or without intact insulin signaling in the liver as compared with mice receiving saline (Figure 1, A and B, $P=2.56 \mathrm{E}-07$ ). Thus, FGF21 was as potent at reducing body weight in mice lacking insulin action in the liver as it was in controls.

Together with increased body weight, we found that liver weight was increased by $10 \%$ in control mice on an HFD (Figure 1C). As previously described, FGF21 reduced liver weight by $18 \%$ in control animals on a CD and by $23 \%$ in control animals on an HFD. In LIRKO animals, which displayed $18 \%$ smaller livers than controls on a $\mathrm{CD}$, we observed a $17 \%$ increase in liver weight in those on an HFD (Figure 1C). FGF21 had no effect on liver weight in mice on a $\mathrm{CD}$ and reduced liver weight by $15 \%$ in LIRKO animals on an HFD. Dual-energy x-ray absorptiometry (DXA) analysis performed on the HFD-fed groups of mice showed that FGF21 induced a slight reduction in lean mass in control mice $(22.1 \pm 0.7 \mathrm{~g}$ and $20.3 \pm 0.5 \mathrm{~g}$ in saline-treated and FGF21-treated control mice, respectively) (Figure 1D). DXA showed significantly reduced fat mass in control mice $(9.4 \pm 0.6 \mathrm{~g}$ and $6.4 \pm 0.6 \mathrm{~g}$ in saline-treated and FGF21treated control mice, respectively) (Figure 1E), as well as reduced adiposity ( $29.7 \pm 1.4 \%$ and $24.1 \pm 1.4 \%$ in saline-treated and FGF21treated control mice, respectively) (Supplemental Figure 2A). We observed a similar reduction in lean mass induced by FGF2 1 in LIRKO animals on an HFD, even though the effect was less pronounced (lean mass: $20.6 \pm 0.6 \mathrm{~g}$ and $19.3 \pm 0.4 \mathrm{~g}$ in saline-treated and FGF21-treated LIRKO mice, respectively) (Figure 1D). We also observed a reduction in fat mass and adiposity in LIRKO mice following FGF21 administration (fat mass: $9.1 \pm 0.8 \mathrm{~g}$ and $7.9 \pm 0.3 \mathrm{~g}$ [Figure 1E] and adiposity: $30.4 \pm 1.9 \%$ fat and $29.1 \pm 0.8 \%$ fat [Supplemental Figure 2A] in saline-treated and FGF21-treated LIRKO mice, respectively). Interestingly, LIRKO mice gained less adipose tissue following an HFD than did controls. Indeed, we observed a 2.5 -fold increase in perigonadal and s.c. fat pads weights following an HFD in controls compared with a 1.5 -fold increase in both depots in LIRKO mice, although this did not reach statistical significance. FGF21 administration had no significant effect on fat pad weights in any of the mice groups (Supplemental Figure 2, $\mathrm{B}$ and $\mathrm{C}$ ). Despite the minor quantitative differences in the ability of FGF2 1 to reduce fat mass in control and LIRKO mice (mainly due to the fact that LIRKO mice are less prone to gain fat on an HFD), FGF21 was equally effective in reducing body weight in both control and LIRKO mice, indicating that LIRKO mice had a normal response to FGF21 treatment.

FGF21 reversed byperglycemia and byperinsulinemia and improved whole-body insulin sensitivity independently of insulin action in the liver. A major effect of FGF21 is to improve glycemic control in obese mice $(17,26)$. In control mice, fed glucose levels were moderately increased by HFD feeding $(158.7 \pm 8.0 \mathrm{mg} / \mathrm{dl}$ vs. $185.2 \pm 9.0 \mathrm{mg} / \mathrm{dl}$ on a CD and HFD, respectively), however, FGF21 treatment for 14 days reduced glucose levels in control HFD mice by $23 \%$ from $185.2 \pm 9.0 \mathrm{mg} / \mathrm{dl}$ to $142.6 \mathrm{mg} / \mathrm{dl}(P=0.0026)$ (Figure $2 \mathrm{~A})$. Insulin levels were also normalized upon FGF21 treatment in the control cohort (Figure $2 \mathrm{~B}$ ). In control mice, fed insulin levels were reduced from $2.1 \pm 0.7 \mathrm{ng} / \mathrm{ml}$ to $0.3 \pm 0.1 \mathrm{ng} / \mathrm{ml}$ following FGF2 1 administration $(P=0.038)$ and from $2.4 \pm 0.3 \mathrm{ng} / \mathrm{ml}$ to $0.7 \pm 0.1 \mathrm{ng} / \mathrm{ml}$ in control HFD animals $(P=0.016)$, suggesting an improvement in insulin sensitivity with FGF21. 

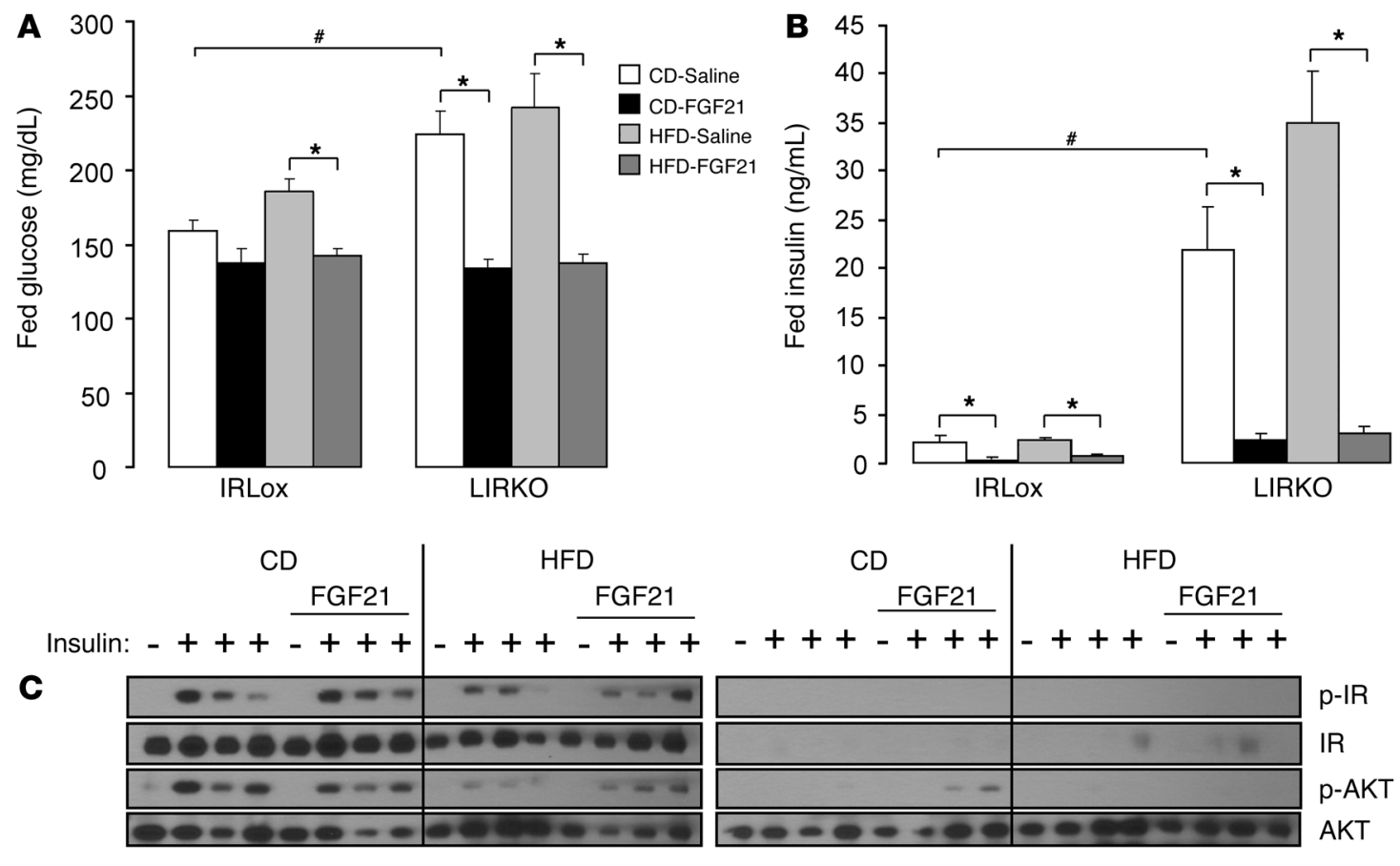

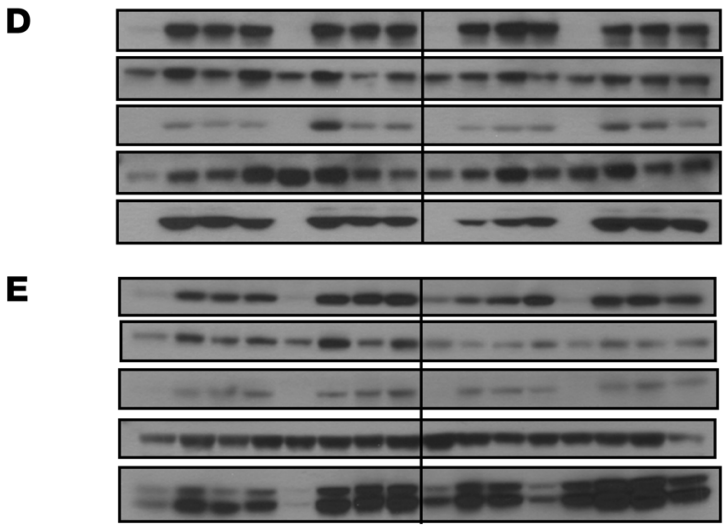

IRLox
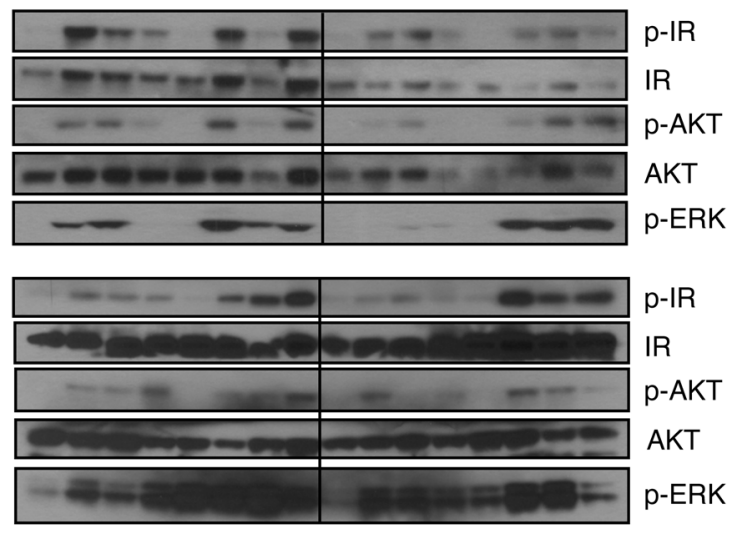

LIRKO

\section{Figure 2}

Glycemia, insulinemia, and insulin signaling in mice treated with FGF21. Control and LIRKO mice on a CD or an HFD for 7 weeks were treated with saline or FGF21 (1 mg/kg/day) delivered s.c. by osmotic pump during the last 2 weeks of the diet. Metabolic parameters (A and B) and insulin signaling (C-E) were determined on day 14 after pump insertion. Data represent the means \pm SEM. $P$ values were determined by nonparametric statistical tests. $\# P<0.05$ between genotypes; ${ }^{*} P<0.05$ with FGF21 treatment. White bars represent saline-treated mice on a CD, black bars represent FGF21-treated mice on a CD, light gray bars represent saline-treated mice on an HFD, and dark gray bars represent FGF21-treated mice on an HFD. (A) Fed glucose was reduced by $40 \%$ with FGF21 treatment $(223.5 \pm 16.1 \mathrm{mg} / \mathrm{dl}$ to $133.7 \pm 5.9 \mathrm{mg} / \mathrm{dl})$ in LIRKO mice on a CD and by $44 \%(242.8 \pm 22.0 \mathrm{mg} / \mathrm{dl}$ to $137.7 \pm 5.3 \mathrm{mg} / \mathrm{dl})$ in LIRKO mice on an HFD. (B) Administration of FGF21 to LIRKO mice reduced insulin levels from $21.8 \pm 4.5 \mathrm{ng} / \mathrm{ml}$ to $3 \pm 0.7 \mathrm{ng} / \mathrm{ml}$ in mice on a CD and from $35 \pm 5.4 \mathrm{ng} / \mathrm{ml}$ to $3 \pm 0.7 \mathrm{ng} / \mathrm{ml}$ in mice on an HFD. $n=10-20$ animals per group. (C-E) Insulin signaling in vivo. Data shown are for 4 mice in each group of 5 to 7 animals. Total lysates obtained from liver (C), skeletal muscle (D), or s.c. adipose tissue (E) from control or LIRKO mice on a CD or an HFD and treated with saline or FGF21 before insulin injection were immunoblotted with various antibodies against insulin signaling molecules as indicated.

We found that LIRKO mice exhibited fed hyperglycemia $(223.5$ $\pm 16.1 \mathrm{mg} / \mathrm{dl}, P=0.014)$ due to the inability of insulin to regulate hepatic glucose output (28), and this did not significantly worsen on an HFD $(242.8 \pm 22.0 \mathrm{mg} / \mathrm{dl})$. LIRKO mice also displayed marked hyperinsulinemia, reflecting compensation by the pancreatic $\beta$ cell to overcome liver insulin resistance and decreased insulin clearance by the liver (24). Indeed, insulin levels were $21.8 \mathrm{ng} / \mathrm{ml}$ in
LIRKO animals on a CD and rose to $35 \mathrm{ng} / \mathrm{ml}$ on an HFD. Remarkably, and despite the absence of insulin signaling in the liver, we found that hyperglycemia and hyperinsulinemia in LIRKO mice were dramatically improved upon FGF21 treatment (Figure 2, A and B): fed glucose was reduced by $40 \%$ in LIRKO mice on a CD $(P=0.0001)$ and by $44 \%$ in LIRKO mice on an HFD $(P=0.0002)$. These results indicate that the glucose-lowering effect of FGF21 is 
independent of insulin action in the liver and is thus independent of hepatic glucose output. Likewise, chronic FGF21 administration reduced insulin levels by $86 \%$ in LIRKO mice on a CD $(P=0.005)$ and by greater than $90 \%$ in mice on an $\operatorname{HFD}(P=0.004)$.

Reduced glucose levels in response to challenge with an i.p. injection of insulin were slightly improved with chronic FGF21 treatment in both control and LIRKO mice on a CD $(P=0.028$; Supplemental Figure 3A). When mice were fed an HFD, FGF21 improved insulin tolerance tests in both control and LIRKO mice $(P=0.002$; Supplemental Figure 3, B and C), confirming previous studies showing improvement in insulin sensitivity in obese mice treated with FGF21 $(17,26)$.

To further examine the effects of FGF21 on insulin sensitivity, we investigated insulin signaling following insulin injection into the inferior vena cava in vivo. In the livers of control mice on a $\mathrm{CD}$, insulin stimulated tyrosine phosphorylation of the IR and phosphorylation of AKT, and this was unchanged upon FGF21 treatment (Figure 2C). As expected, when we placed the mice on an HFD, both IR levels and insulin-stimulated tyrosine phosphorylation were decreased, indicating insulin resistance in this tissue (Figure 2C). Likewise, in HFD-fed mice, insulin-stimulated AKT phosphorylation was decreased (Figure 2C). We observed that FGF21 treatment was able to partially restore insulin-induced tyrosine phosphorylation of the IR and phosphorylation of AKT (Figure 2C and Supplemental Figure 4, A and B). These data suggest that, in control mice, FGF21 treatment was able to only partially rescue insulin signaling in the liver when mice were exposed to an HFD. As expected, in LIRKO mice, there was no tyrosine phosphorylation of the IR or the IGF1 receptor following insulin stimulation, and there were also no detectable downstream insulin signaling events (Figure 2C). In skeletal muscle and in adipose tissue, insulin induced phosphorylation of the IR, AKT, and ERK in both control and LIRKO mice, and this was enhanced by FGF2 1 in both CD- and HFD-fed control and LIRKO animals (Figure 2, D and E, and Supplemental Figure 4, C-J).

FGF21 did not prevent gluconeogenesis in LIRKO animals, but increased glucose uptake in adipose tissue. Previous studies suggested that the ability of FGF21 to lower glucose in obese mice was due to increased insulin sensitivity in the liver and a concomitant reduction in hepatic glucose output (27). Our data showing a reduction in blood glucose induced by FGF21, even in mice lacking IRs in the liver, suggested that this may not be the case, so we investigated whether FGF21 itself is able to reduce hepatic glucose output by performing a pyruvate challenge test. In control mice on either a CD or an HFD, FGF21 was unable to reduce glucose output following an i.p. injection of pyruvate (Figure 3A and Supplemental Figure 5, A-C). In LIRKO mice, basal glucose output following pyruvate challenge was enhanced as compared with both CD-fed or HFD-fed controls, reflecting liver insulin resistance; in LIRKO mice, FGF21 administration further increased this response (Figure $3 \mathrm{~A}$ and Supplemental Figure 5, A-C). These results were concordant with the gene expression profile we observed in the liver. The expression of Ppargc1a, which is known to activate metabolic pathways linked to the fasted response in the liver, was increased by 2 -fold and 2.3-fold, respectively, in CD- and HFD-fed control mice that were treated with FGF21 (Figure 3B). The expression of G6pc, encoding glucose-6-phosphatase, a key enzyme in gluconeogenesis and glycogenolysis, was reduced by $40 \%$ with FGF21 treatment in the livers of control mice on a CD, but not in those on an HFD. On the other hand, the expression of Pck1, encoding the rate-limiting enzyme in gluconeogenesis phosphoenolpyruvate carboxykinase, tended to be increased by FGF21 treatment in CD- and HFD-fed control mice (Figure 3B). In the livers of LIRKO mice, expression levels of Ppargc1a, G6pc, and Pck1 were elevated by 4-fold, 3-fold, and 1.4-fold, respectively, compared with those in control mice on a CD and by 4-fold, 4.4-fold, and 2-fold, respectively, compared with those in control mice on an HFD (Figure 3B). More importantly, we found that in the livers of LIRKO mice, FGF21 did not regulate the expression of these genes, regardless of diet (Figure 3B). These data indicate that the program of gluconeogenesis was enhanced in LIRKO mice livers due to the lack of insulin action and that FGF21 was unable to correct this defect. The liver glycogen content was reduced by FGF21 in CD- and HFD-fed control mice (Supplemental Figure 5D). In LIRKO mice, as previously reported, glycogen content was decreased as compared with that in CD- and HFD-fed control mice, and this was further decreased by the addition of FGF21 (Supplemental Figure 5D). Therefore, altogether, these results suggest that FGF21 lowers glucose levels independently of hepatic glucose metabolism.

To identify the tissue(s) responsible for the glucose-lowering effect of FGF21, we performed in vivo glucose uptake experiments by measuring the accumulation of 2-deoxy-D-[1, 2-C $\left.{ }^{14}(\mathrm{~N})\right]$ glucose in various tissues following insulin stimulation. As expected, in control mice, insulin induced a 3 -fold increase in glucose uptake in skeletal muscle, an 8-fold increase in interscapular BAT (iBAT), a 4-fold increase in s.c. adipose tissue, and a 2-fold increase in perigonadal adipose tissue (Figure 3, C and D, and Supplemental Figure 5, E and F, open bars). In mice treated with FGF21, glucose uptake following insulin stimulation was slightly diminished in these tissues (Figure 3, C and D, and Supplemental Figure 5, $\mathrm{E}$ and F, black bars), indicating that FGF21 was unable to further enhance insulin-stimulated glucose uptake in these tissues. However, in the basal state without insulin stimulation, FGF21 itself induced a 2 -fold increase in glucose uptake in iBAT of control mice (Figure 3D) and a 1.3-fold increase in glucose uptake in perigonadal and s.c. WAT (Supplemental Figure 5, E and F). In LIRKO animals, insulin-induced glucose uptake was similar in skeletal muscle and adipose tissue (Figure 3, C and D, and Supplemental Figure 5, E and F, white bars), and FGF21 had a very moderate effect on insulin-stimulated glucose uptake in these tissues. As in the control mice, in the absence of insulin stimulation, FGF21 induced an even larger (3-fold) increase in glucose uptake in BAT of LIRKO mice (Figure 3D), which correlated with higher expression levels in this tissue of Slc2a1, the gene encoding GLUT1 (Supplemental Figure 6), but had no effect on, or even diminished, glucose uptake in skeletal muscle and other tissues (Figure 3C and Supplemental Figure 5, E and F). Thus, FGF21 increased basal glucose uptake in fat, especially BAT, suggesting that BAT is at least partly responsible for the improved glycemia in FGF21-treated control and LIRKO mice.

FGF21 induced activation of BAT and increased energy expenditure in LIRKO animals. BAT plays a major role in the regulation of energy homeostasis by dissipating energy in the form of heat (29), and FGF21 has been proposed to induce brown fat activation (13). We therefore investigated the effect of FGF21 on energy expenditure in HFD-fed control and LIRKO mice in metabolic cages. We found that, as previously reported (26), FGF21 induced a striking increase in energy expenditure in both groups of mice, with a $46 \%$ increase in $\mathrm{O}_{2}$ consumption during the light cycle and a $55 \%$ increase during the dark cycle in control animals (Figure 4A and Supplemen- 


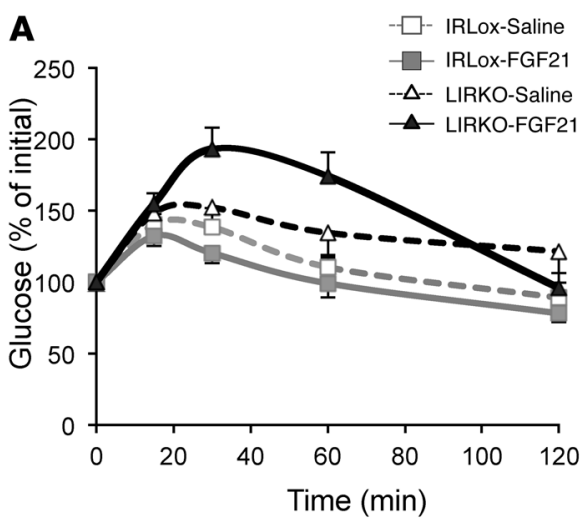

C

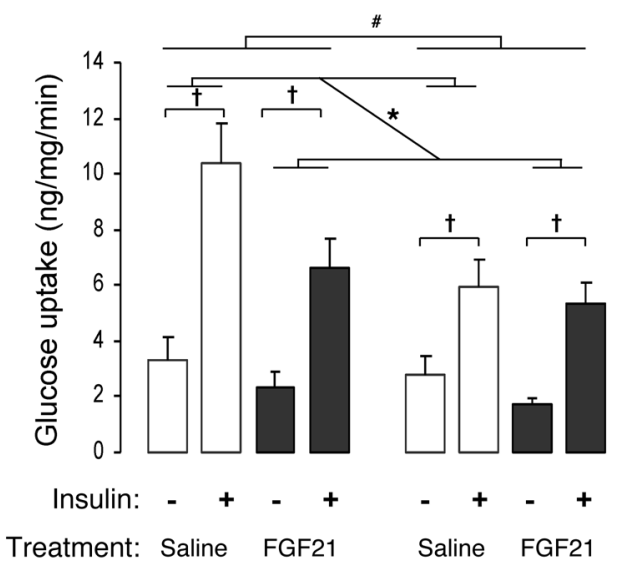

IRLox

LIRKO
B

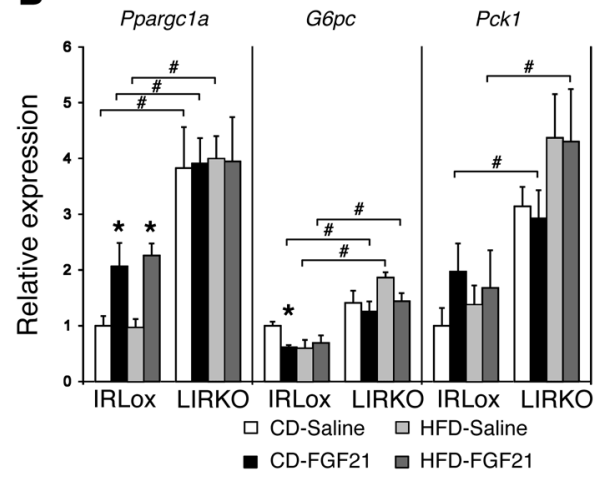

D

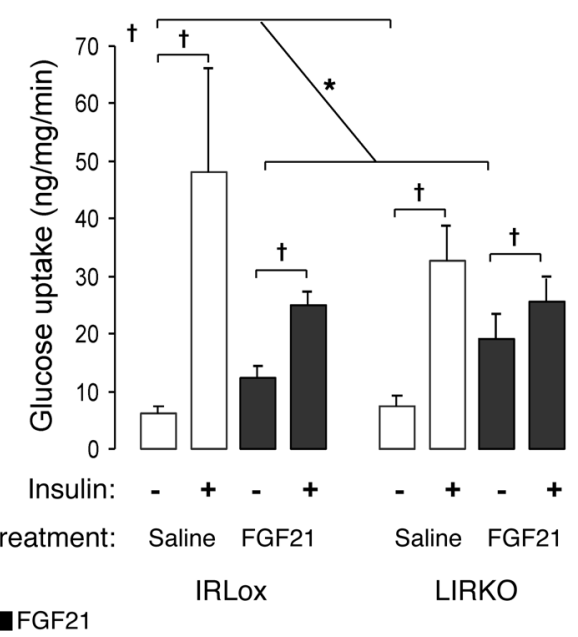

Figure 3

Effect of FGF21 on gluconeogenesis and glucose uptake in skeletal muscle and BAT. Control and LIRKO mice on a CD or an HFD for 7 weeks were treated with saline or FGF21 (1 mg/kg/day) delivered s.c. by osmotic pump during the last 2 weeks of the diet. (A) Pyruvate challenge test. Gray lines with squares represent control mice; black lines with triangles represent LIRKO mice; dashed lines represent saline-treated mice; solid lines represent FGF21-treated mice. (B) Gene expression in liver was assessed by real-time qPCR. $P$ value was determined by nonparametric tests. White bars represent saline-treated mice on a $C D$, black bars represent FGF21-treated mice on a CD, light gray bars represent saline-treated mice on an HFD, and dark gray bars represent FGF21-treated mice on an HFD. (C and D) In vivo glucose uptake was assessed by measuring $\left[{ }^{14} \mathrm{C}\right] \mathrm{DOG}$ uptake in (C) skeletal muscle and (D) iBAT, with or without insulin stimulation, in animals on a CD. $P$ value for $\mathbf{D}$ was determined by 3-way ANOVA. White bars represent saline treatment; black bars represent FGF21 treatment. Data represent the means \pm SEM. ${ }^{*}<0.05$ between genotypes; ${ }^{*} P<0.05$ with FGF21 treatment; $\$ P<0.05$ upon insulin stimulation. $n=6-12$ animals per group.

tal Figure 7A) and a 48\% increase during the light cycle and a $52 \%$ increase during the dark cycle in LIRKO animals (Figure 4B and Supplemental Figure 7A). We observed similar results with $\mathrm{CO}_{2}$ production (Supplemental Figure 7, B and C). FGF21 induced a $45 \%$ increase in $\mathrm{CO}_{2}$ production during the light cycle and a $54 \%$ increase during the dark cycle in control animals and a $47 \%$ increase during the light cycle and a $52 \%$ increase during the dark cycle in LIRKO animals. We found that the respiratory exchange ratio was approximately 0.76 in all groups, indicating no change in the fuel sources used. The increased energy expenditure induced by FGF21 was not due to increased activity (Supplemental Figure 7D), and food intake was similarly increased by FGF21 in both control and
Slc2a1, Lipe, Ucp1, Ucp2, and Ucp3 in control and LIRKO mice. Furthermore, we observed the appearance of multilocular adipocytes in s.c. white fat following FGF21 treatment in control mice on a CD, indicating a browning of s.c. adipose tissue (Supplemental Figure 8B). The appearance of multilocular adipocytes in response to prolonged FGF21 exposure was similar in LIRKO mice on a CD and was conserved in those on an HFD, although the number of multilocular cells appeared to be reduced (Figure 4D). Increased multilocular adipocytes following FGF21 treatment was correlated with an increase in Ucp 1 mRNA expression, a marker of thermogenesis in brown adipocytes, in s.c. adipose tissue in both control and LIRKO mice (Figure 4E). Indeed, FGF21 increased Ucp1 expression by 100-fold 

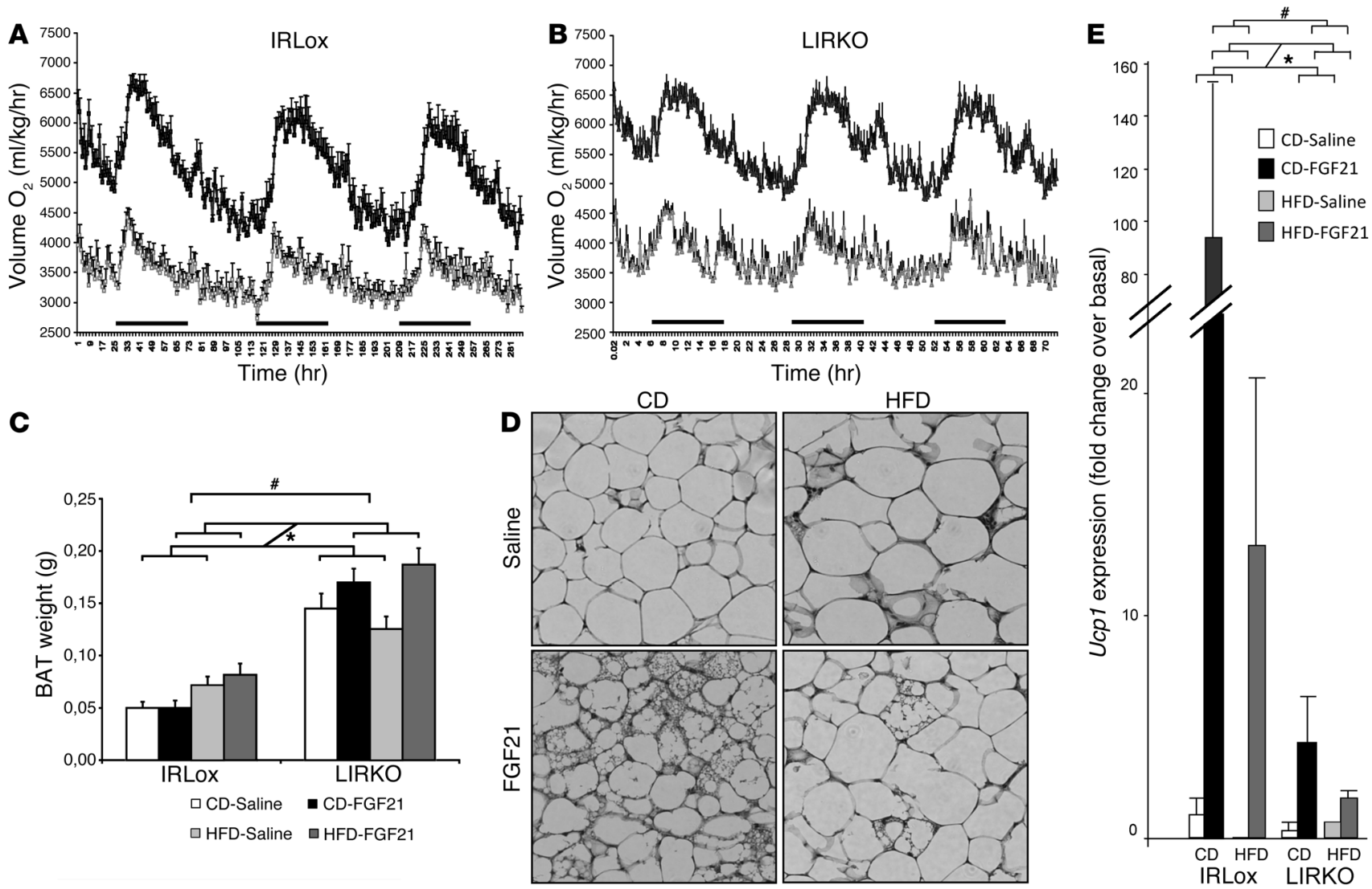

Figure 4

Energy homeostasis and BAT activation following chronic FGF21 treatment. Control and LIRKO mice on a CD or an HFD for 7 weeks were treated with saline or FGF21 (1 mg/kg/day) delivered s.c. by osmotic pump during the last 2 weeks of the diet. CLAMS analysis was determined for 3 days, starting on day 7 after insertion of the pumps. (A and $\mathbf{B}) \mathrm{O}_{2}$ consumption in control (A) and LIRKO (B) animals on an HFD. Gray lines represent saline-treated mice; black lines represent FGF21-treated mice. (C) BAT weight. White bars represent saline-treated mice on a CD, black bars represent FGF21-treated mice on a CD, light gray bars represent saline-treated mice on an HFD, and dark gray bars represent FGF21-treated mice on an HFD. (D) H\&E staining of s.c. adipose tissue sections from LIRKO mice. Original magnification, $\times 40$. (E) Ucp1 expression in s.c. adipose tissue determined by qPCR. Data represent the means \pm SEM. $P$ values were determined by 3-way ANOVA analysis.

in control mice on a CD and by more than 10 -fold in control mice on an HFD. In LIRKO mice, FGF21 induced a 4-fold increase in Ucp1 expression in mice on a CD and a 2-fold increase in those on an HFD. Altogether, our results support the idea that FGF21 increases the mass and activity of adipose tissue involved in the dissipation of energy (both BAT and browning of WAT), and this likely contributes to the blood glucose-lowering effect of FGF21.

FGF21 potency in mice without $i B A T$. To further investigate the role of BAT in mediating the effect of FGF21 on body weight, energy expenditure, and glucose homeostasis, we surgically removed the iBAT, which represents greater than $60 \%$ of constitutive BAT. For control mice on an HFD, with or without iBAT, we administered the same FGF21 treatment as described above and implanted them with a minipump at the time of surgery. We found that FGF21 was equally effective in reducing body weight in mice with or without iBAT (Figure 5A). This was accompanied by a similar reduction in blood glucose levels in both groups, which was statistically significant 1 week after surgery (Figure 5B). The high energy expenditure produced by FGF21 infusion, as measured by $\mathrm{O}_{2}$ consumption (Figure $5 \mathrm{C}$ ) or $\mathrm{CO}_{2}$ production (Supplemental Figure 9A), was also unaffected by the removal of iBAT. There was no change in activity between the two groups, and we observed an increase in food intake in the mice without iBAT (Supplemental Figure 9, B and C). The reduction in liver weight induced by FGF21 in the sham group was similar to that observed in the previous cohort (Supplemental Figure 9D), and this effect was lost in the group of mice in which iBAT had been removed. In addition, the white fat pads (both perigonadal and s.c.) tended to be smaller in the group of mice without iBAT compared with those of the sham control group (Supplemental Figure 9D), suggesting that browning of white fat is increased following the surgical removal of iBAT. Gene expression analysis showed a tendency toward increased beige adipogenesis in s.c. fat, with a slightly increased expression of the master regulator of adipogenesis Pparg and other typical beige fat markers such as Ppargc1a, Prdm16, and Cidea (Figure 5D). Somewhat surprisingly, however, Ucp1 expression in WAT followed an opposite pattern, i.e., it tended to decrease following iBAT removal, although this change was not statistically significant due to the large variability between animals (Figure 5D). Taken together, these results indicate that removal of the major constitutive brown fat pad does not impede FGF21 action, suggesting that either the remaining depots of constitutive BAT (Supple- 
A
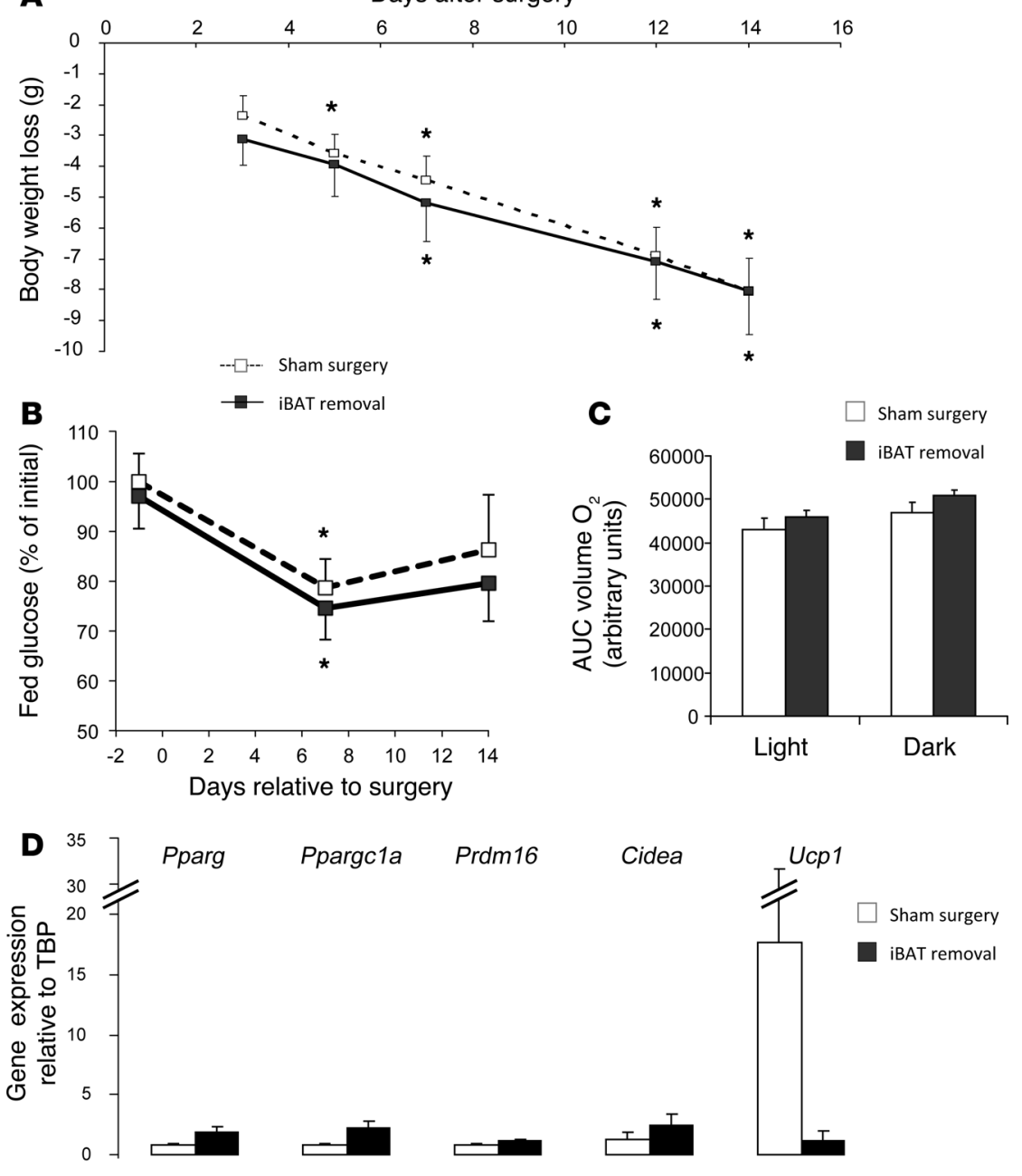

\section{Figure 5}

FGF21 is still potent in mice lacking iBAT. Control mice on an HFD for 12 weeks, with or without iBAT, were treated with FGF21 (1 mg/kg/day) delivered s.c. by osmotic pump during the last 2 weeks of the diet. Data represent the means \pm SEM. $n=6-7$ animals per group. ${ }^{*} P<0.05$. (A) Weight loss following surgery and FGF21 treatment. $P$ value was determined by 1 -way ANOVA. (B) Glucose levels are represented as a percentage of initial glucose before surgery. Dashed lines represent animals with sham surgery; solid lines represent animals with surgical removal of iBAT. $P$ value was calculated by a Student's $t$ test. (C) $\mathrm{O}_{2}$ consumption per animal measured by CLAMS. White bars represent mice with sham surgery, and black boxes represent mice without iBAT, during light or dark cycles. $P$ value was calculated by a Student's $t$ test. (D) Gene expression in s.c. adipose tissue was assessed by real-time qPCR. White bars represent mice with sham surgery; black bars represent mice without iBAT. $P$ value was calculated by a Student's $t$ test. mental Figure 9D) or the browning of white fat is sufficient to mediate FGF2 1 effects.

Regulation of lipid metabolism by FGF21 in the liver requires insulin action. Among the beneficial actions of FGF21, treatment with FGF21 improves the blood lipid profile and reduces hepatosteatosis in obese animals $(17,26)$. In contrast to its action on glucose levels, we found that the ability of FGF21 to lower plasma lipid concentrations was compromised in LIRKO mice. Thus, while we found that plasma cholesterol levels were reduced by $33 \%$ in FGF21-treated control mice on a CD and by $23 \%$ in control mice on an $\operatorname{HFD}(P=0.007)$, plasma cholesterol levels were not reduced in mice with IR deletion in the liver $(P=0.136)$ (Figure 6A). Further, we observed that plasma FFA and triglyceride levels were not affected by FGF21 (plasma levels of FFAs and triglycerides had a nonsignificant FGF21-induced reduction of $38 \%$ and $28 \%$, respectively, in CD-fed control mice, but not in HFD-fed control mice; Supplemental Figure 10, A and B). In LIRKO mice, circulating FFA levels were reduced by $50 \%$ compared with those in control mice on a CD, and there was no regulation by FGF21 (Supplemental Figure 10A). Triglycerides levels in CD- or HFD-fed LIRKO mice were similar to those in control mice, and again, there was no effect of FGF21 treatment (Supplemental Figure 10B). As expected, FGF21 infusion decreased liver triglyceride content in control mice on a CD and in the livers of control mice on an HFD, where larger amounts of triglyceride were stored at basal $(P=0.03)$ (Figure 6B). Remarkably, in LIRKO mice, which already had lower liver triglyceride levels than controls in the basal state, this FGF21 effect was lost $(P=0.838)$ (Figure 6B). In addition, we found that the removal of iBAT in mice on an HFD had no effect on liver triglyceride content, with or without FGF21 treatment (Supplemental Figure 10C). These data indicate that liver insulin signaling is required for FGF21 action on lipid metabolism.

Our analysis of the expression of key genes involved in the regulation of hepatic metabolism in the livers of mice exposed to FGF21 revealed that most of the gene regulation by FGF21 in this tissue was absent in LIRKO mice (Figure 6C). In the heatmap representation shown in Figure 6C, two groups of genes were created according to their regulation by FGF21 in control mice on a CD: genes upregulated by FGF21 (Figure 6C, upper panel), and genes repressed by FGF21 (Figure 6C, bottom panel). Interestingly, we observed a similar pattern of gene expression for these two particular sets of genes in the livers of LIRKO mice under basal conditions. The genes that were upregulated (Lepr, Igfbp2, and Abcg5) or downregulated ( $S c d 1, P k l r$, and Gck) by FGF21 exposure in the livers of control mice were similarly up- or downregulated in the livers of LIRKO mice before FGF21 treatment, and FGF21 failed to further 
A

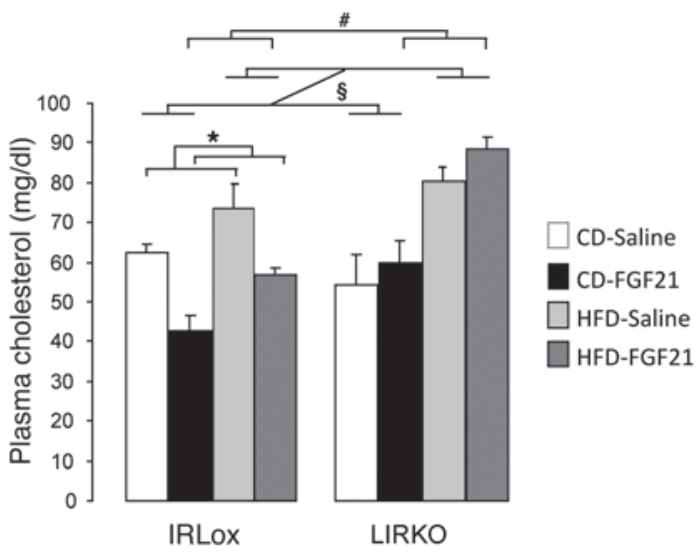

C
B

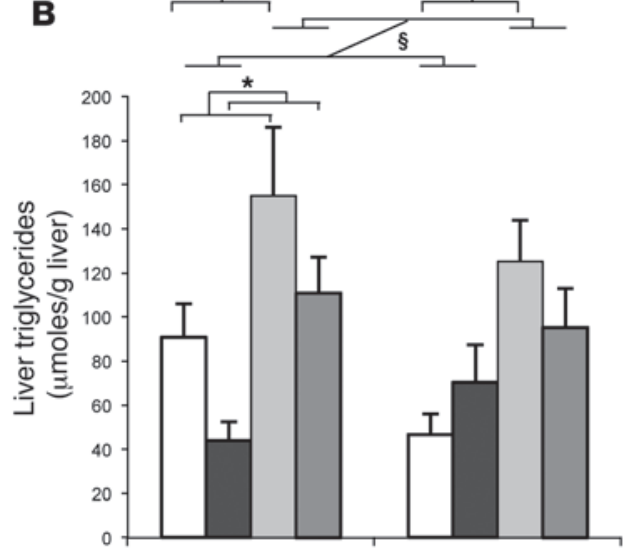

IRLox
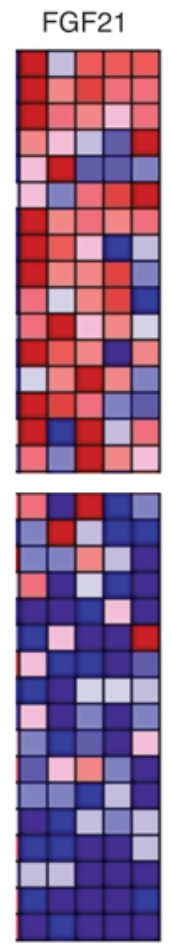

\section{LIRKO}

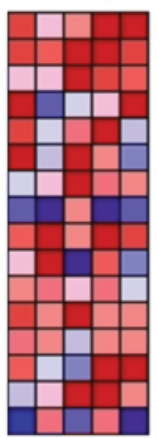

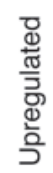

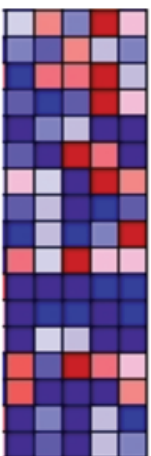

\begin{abstract}
Saline
\end{abstract}
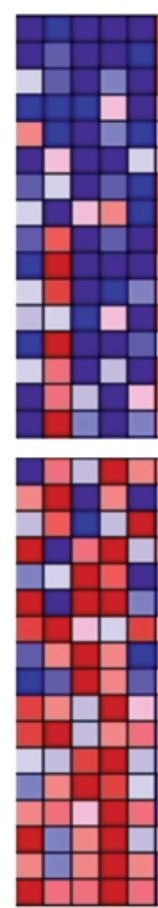

\section{Figure 6}

Lipid homeostasis and liver gene expression following FGF21 treatment. Control and LIRKO mice on a CD or an HFD for 7 weeks were treated with saline or FGF21 (1 $\mathrm{mg} / \mathrm{kg} /$ day) delivered s.c. by osmotic pump during the last 2 weeks of the diet. (A) Plasma cholesterol levels in the fed state. (B) Liver triglyceride content. Data represent the means \pm SEM. $P$ value was calculated using 2-way or 3-way ANOVA. ${ }^{\#} P<0.05$ between genotypes; $\S P<0.05$ between diets; ${ }^{*} P<0.05$ with FGF21 treatment. White bars represent saline-treated mice on a $\mathrm{CD}$, black boxes represent FGF21-treated mice on a $C D$, light gray bars represent saline-treated mice on an HFD, and dark gray bars represent FGF21-treated mice on an HFD. $n=5$ to 11 animals per group. (C) Liver gene expression. The expression of selected genes was measured by GPCR, and heatmaps were generated using GenePattern software. Red color indicates upregulation; blue color indicates downregulation. Each lane represents an individual mouse. regulate the expression of these genes. Thus, with regard to these sets of genes, deletion of the IR mimics the FGF21 effect in control animals. For example, the upregulation we observed of $A b c g 5$ and $A b c g 8$ genes, transporters involved in cholesterol excretion, in the livers of control mice on a CD in response to FGF21 was absent in LIRKO animals on either a CD or an HFD, which displayed higher expression levels of these two cholesterol transporters at basal levels (Supplemental Figure 11A). Likewise, the expression of Ppargc1a and Cpt1a, a gene involved in the regulation of long-chain fatty acid transport to the mitochondria, was higher in LIRKO animals (Supplemental Figure 11A). Similarly, the FGF21-induced downregulation of genes involved in lipid synthesis such as Gck, Pklr, and Scd1, which was observed in the livers of control animals, was reduced in the livers of LIRKO mice, partly because the basal expression of these genes was already markedly decreased (Supplemental Figure $11 \mathrm{~B})$. These results indicate that FGF21 regulation of the expres- sion of genes involved in lipid metabolism occurs, at least in part, through the modulation of insulin action in this tissue.

\section{Discussion}

FGF21 is an important regulator of carbohydrate and lipid metabolism and is a promising drug candidate for the treatment of metabolic diseases, as it reverses several metabolic syndrome-associated defects at once. Indeed, FGF21 reduces the weight and adiposity of obese animals, as well as hepatosteatosis, hyperglycemia, and dyslipidemia, and thus is associated with enhanced insulin sensitivity $(17,26)$. Since obesity and insulin sensitivity are tightly correlated, it is difficult to distinguish the antiobesity and antidiabetic properties of FGF21.

Previous studies have suggested that part of the mechanism of action of FGF21 occurs through the restoration or enhancement of insulin sensitivity, especially in the liver (27). Also, pharmaco- 
logical doses of FGF21 reduce glucose levels without triggering hypoglycemia, suggesting that FGF21 acts as an insulin sensitizer rather than as an insulin mimetic (7). In the present study in LIRKO mice, we demonstrate that FGF21 is able to ameliorate obesity and improve glucose homeostasis in diabetic mice, despite a total absence of insulin signaling in the liver. This occurred without any reduction of hepatic glucose output, indicating that FGF21 is able to regulate glucose metabolism independently of hepatic insulin action on gluconeogenesis. Our results also show that FGF21 action on lipid metabolism requires functional insulin signaling in the liver. These conclusions are further supported by recent findings demonstrating that deletion of FGFR1 in adipose tissue abrogates the glycemic effects of FGF21, while the FFA effects remain intact (18).

FGF21 can normalize hyperglycemia in lean or obese diabetic mice with IR deletion in the liver via two possible mechanisms: (a) FGF21 reduces glucose output independently of insulin, and/or (b) FGF21 lowers plasma glucose levels through its action on other organs/tissues. Gene expression studies, as well as pyruvate tolerance tests, revealed that reduced gluconeogenesis is not necessary for FGF21 to normalize blood glucose. Rather, it appears that activation of BAT and perhaps its effects on glucose uptake in other tissues are critical factors in mediating the effects of FGF21 on carbohydrate metabolism. These data appear to contradict previous studies showing that in obese ob/ob mice, FGF21 reduces hepatic output during hyperinsulinemic clamp, without any modification of glucose clearance by skeletal muscle or adipose tissue (27). However, in the latter study, the contribution of glucose uptake in BAT was not evaluated, and leptin deficiency itself in ob/ob mice may be a contributing factor to this difference. Therefore, despite the ability of FGF21 to increase insulin sensitivity and reduce hepatic glucose output, these events are not required to reestablish normal glucose control as long as other organs, such as WAT or BAT, can compensate for this lack of action. In accordance with this, it was previously reported that chronically delivered FGF21 not only improves the ability of insulin to inhibit hepatic glucose output in DIO mice, but it also enhances insulin-stimulated glucose uptake in muscle and BAT (17). Also, the glucose-lowering effect of FGF21 specifically in this tissue is abolished in mice deficient for FGFR1, the main receptor for FGF21, in adipose tissue (18) and is also abolished in lipoatrophic mice (30), further confirming the importance of this tissue in mediating FGF21 action on carbohydrate metabolism. The insulin-independent glucose-lowering effect of FGF21 may provide a therapeutic advantage in advanced cases of type 2 diabetes, in which $\beta$ cell function is severely impaired and insulin becomes limiting. This is in contrast to insulin-sensitizing compounds such as TZDs and metformin, which require some insulin action in the liver to efficiently improve glucose tolerance (31). FGF21 also controls insulin secretion by improving $\beta$ cell function and protecting it from apoptosis during obesity (32).

Our data suggest that activation of BAT and the browning of white fat are responsible for the glucose-lowering effect of FGF21 in LIRKO mice at the whole-animal level. This is indicated by both increased glucose uptake in BAT as well as by markedly increased energy expenditure with FGF21 exposure. The exact mechanism of this effect is unknown, but FGF21-augmented glucose uptake in BAT may involve increased GLUT1 expression, as demonstrated in studies of 3T3-L1 adipocytes and FGF21-dosed mice (7) and in studies of BAT from LIRKO mice (Supplemental Figure 6). We observed increased basal glucose uptake in WAT and BAT with
FGF21 treatment, but insulin-driven stimulation of glucose uptake in these tissues was not further enhanced by FGF21. In LIRKO animals, FGF21 may also restore insulin sensitivity and glucose uptake in mice on an HFD, especially in s.c. adipose tissue, and may thereby contribute to an overall improvement in blood glucose levels (not shown). Increased glucose uptake in BAT is paralleled with an activation of this tissue (Supplemental Figure 6) and a dramatic increase in energy expenditure. However, surgical removal of iBAT did not abolish the metabolic effects of FGF21. We also made a similar observation in a small number of LIRKO mice, suggesting that increased metabolism is accountable for FGF21 action to reduce blood glucose (data not shown). Although the remaining and more widely dispersed brown adipocytes may contribute to the residual beneficial effect of FGF21, these results imply that the increased energy expenditure and weight loss induced by FGF21 are not solely dependent on the activation of this constitutive brown fat depot and might involve other tissues. Indeed, we also observed browning of s.c. WAT, as evidenced by the appearance of multilocular adipocytes and increased Ucp1 expression in response to chronic FGF21 exposure (Figure 4, D and E). Similar results were reported earlier (33). The identification of brown fat in humans (34-36) and the fact that this depot can be activated reinforce the interest in FGF21 for use in obese diabetic patients. This increase in energy expenditure would promote a diminution of obesity (37), a feature not common to other therapeutic agents used to treat type 2 diabetes such as thiazolidinediones or insulin, which are associated with weight gain $(38,39)$. The role of FGF21, however, may be more complex, since one recent report suggested that FGF21 KO mice are refractory to both the beneficial and detrimental effects of thiazolidinediones, although this has yet to be confirmed in other FGF21-null strains (40).

In contrast to the normalization of hyperglycemia, which can occur without a direct effect on the liver, it appears that intact hepatic insulin signaling is required for some of the action of FGF21 on lipid metabolism. It also indicates that the liver is a critical target of FGF21 for producing its lipid-lowering action, supported by the findings that neither deletion of FGFR1 in fat (18) nor lipoatrophy (30) impair FGF21 regulation of blood cholesterol levels. Despite weight loss, we found that FGF21 was unable to regulate cholesterol levels in LIRKO animals. The expression of the ABCG5 and ABCG8 transporters that mediate cholesterol efflux is repressed by insulin via inhibition of FOXO1 (25). In LIRKO mice, the expression of these transporters is markedly increased, and as a result, biliary cholesterol secretion is augmented (25). We observed that FGF21 enhanced the expression of these transporters in the liver when the IR was expressed, but had no effect on the livers of LIRKO mice. These data suggest that FGF21 counteracts insulin action on the regulation of these genes. Similarly, we found that the reduction of hepatic triglycerides induced by FGF21 was also largely suppressed in the livers of LIRKO mice. On the other hand, we observed no significant effect of FGF21 on plasma FFA and triglyceride levels in control mice or in LIRKO mice with hepatic insulin resistance. Interestingly, SCD1 is the most FGF21-downregulated gene in the liver and is the main direct target gene in this tissue (18). Scd1 encodes stearoyl-CoA desaturase 1 (SCD1), a microsomal enzyme required for the rate-limiting step in the synthesis of major monounsaturated fatty acids of membrane phospholipids, triglycerides, and cholesterol esters, and appears to be a major regulator of energy homeostasis. Scd1 expression is elevated in various mouse models of obesity, whereas its deletion prevents 


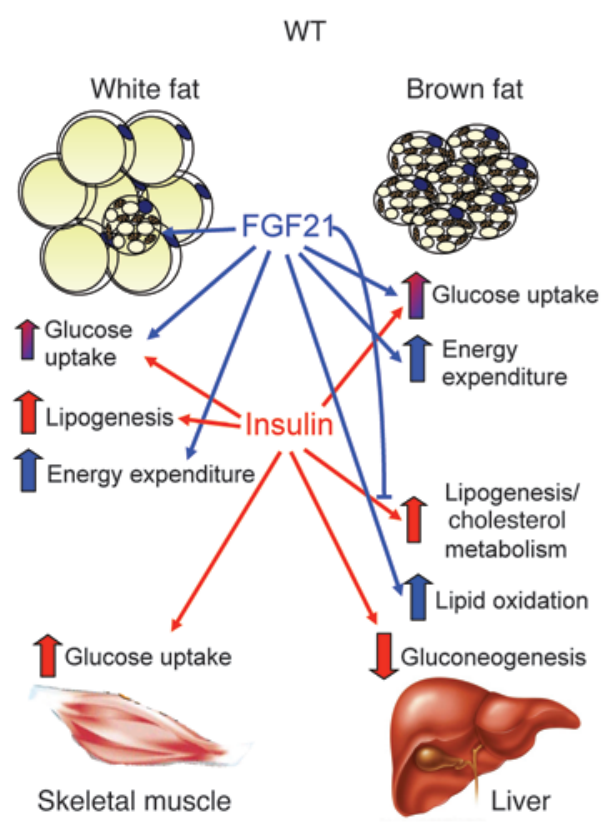

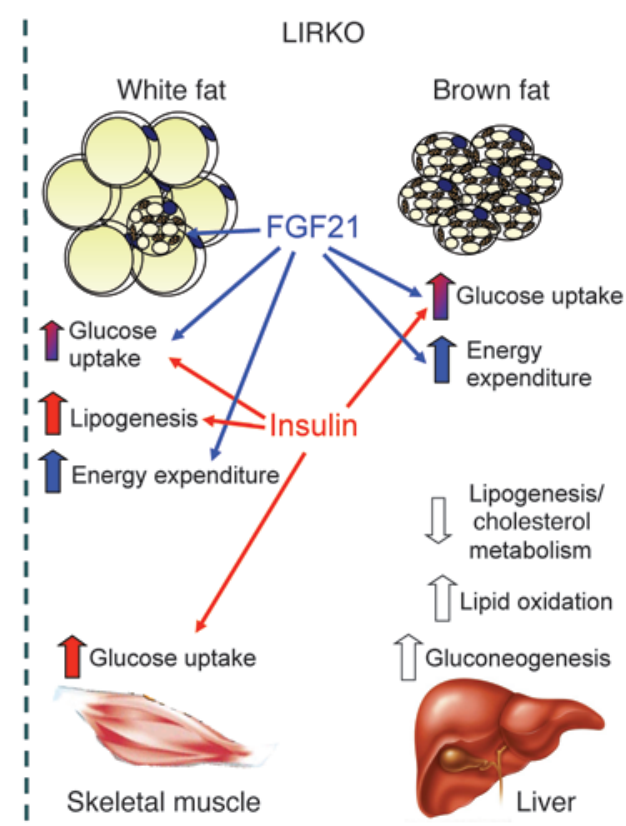

\section{Figure 7}

Crosstalk between insulin and FGF21 for the regulation of metabolism. Red arrows represent metabolic actions controlled by insulin, blue arrows represent metabolic actions regulated by FGF21, and white arrows represent the metabolic outcome induced by deletion of the IR. In LIRKO mice, insulin lost its ability to regulate glucose and lipid metabolism in the liver. In these mice, FGF21 maintained its ability to increase energy expenditure and regulate glucose homeostasis via adipose tissue, but lost its ability to regulate lipid metabolism due to a lack of interaction with insulin in liver.
DIO, hepatosteatosis, and insulin resistance (41-43). One possibility is that SCD1 regulates lipid oxidation by regulating ACC and CPT1 activity. Through its effects on lipid saturation, SCD1 is also an important regulator of membrane fluidity (44). Scd1 expression is dramatically reduced in the livers of LIRKO mice (45), and we found that its regulation by FGF21 was diminished. ACC2 and CPT1a are also regulated by FGF21 (26), which might exacerbate the effect of SCD1, and we found that their regulation was also impaired in LIRKO mice. Together, these results suggest that FGF21 action on lipid metabolism in the liver requires the modulation of insulin action. Interestingly, FGF21 acts in the liver to reduce lipogenesis and enhance lipid oxidation, whereas insulin is well known for its lipogenic action in this organ.

The data from this study reveal a complex crosstalk between FGF21 and insulin-related pathways for the regulation of carbohydrate and lipid metabolism, involving both insulin-dependent and insulin-independent mechanisms (Figure 7). Although some effects of these two factors may be redundant, others appeared to be opposite in nature. We found that both insulin and FGF21 could induce glucose uptake in common as well as in distinct tissues, but they did so using different mechanisms. Whereas insulin induced GLUT4 translocation in skeletal muscle and adipose tissue within minutes, FGF21 appeared to work by increasing GLUT1 expression, and FGF21 acted mainly on glucose uptake in WAT and BAT. Moreover, we observed that FGF21 exerted no additive effect on insulin in vivo and that insulin's effect was maximal and could not be enhanced by continuous FGF21 infusion (Figure 3, C and D, and Supplemental Figure 5, E and F). In the liver, insulin reduced PPARGC1A (46), whereas insulin upregulates SREBP1C, resulting in an enhanced program of lipogenesis $(47,48)$. FGF21, on the other hand, induced PPARGC1A (49) and repressed lipogenesis (50), favoring lipid oxidation (51). These two glucose-lowering compounds also had different properties with regard to weight control $(17,26,39)$. Insulin induced weight gain, whereas FGF21 induced weight loss, suggesting that a combination of the two might be an appropriate approach for the treatment of obesity-associated disorders by enhancing the glucose-lowering efficacy of both FGF21 and insulin, without augmenting the deleterious effects of weight gain. Indeed, if insulin promotes glucose use and storage in the form of triglycerides, FGF21 might compensate for this excessive storage by enhancing glucose clearance via increased energy expenditure.

In summary, FGF21 can normalize glycemia, even in situations of complete hepatic insulin resistance. This feature is likely due to an enhanced metabolic activity of FGF21 in WAT and BAT. FGF21 and insulin act in opposing fashion in the control of genes regulating lipid synthesis and oxidation. These data suggest that FGF21 and insulin, when dosed together, constitute a powerful combination for treating several metabolic disorders including type 2 diabetes.

\section{Methods}

Animals. LIRKO mice were bred as previously described (24). Male control (IRLox) and LIRKO animals were housed in an environmentally controlled facility with a 12-hour light/12-hour dark cycle and had free access to food and water. Mice were fed either a CD containing $22 \%$ of calories derived from fat, $23 \%$ from protein, and 55\% from carbohydrates (Mouse Diet 9F 5020; PharmaServ) or they were fed an HFD containing $60 \%$ of calories derived from fat, $20 \%$ from protein, and $20 \%$ from carbohydrates (OpenSource Diet D12492; Research Diets Inc.) for 7 weeks, starting at the age of $7 \pm 1$ week. Mice were treated with saline or FGF21 $(1 \mathrm{mg} / \mathrm{kg} /$ day of recombinant human FGF21; Eli Lilly) during the last 2 weeks of the 7-week diet using an Alzet osmotic pump (DURECT) inserted s.c. after anesthesia with ketamine/xalazine.

For some experiments, iBAT and the surrounding white fat tissue were removed from the animals. For this, mice were anesthetized with Avertin (tribromoethanol:tert-amyl alcohol, $0.015 \mathrm{ml} / \mathrm{g}$ i.p.), and an incision was made in the interscapular region. The blood vessels entering and leaving the fat pad were ligated and the pads removed. The same procedure was performed for sham surgeries, except that the adipose tissue was maintained. At the time of surgery, these mice were implanted with a minipump delivering FGF21. 
Animal care and study protocols were approved by the Animal Care Committee of the Joslin Diabetes Center and were in accordance with NIH guidelines.

CLAMS and DXA analysis. Comprehensive lab animal monitoring system (CLAMS) and DXA analysis were performed at the Joslin animal core facility. Mice were housed individually in metabolic cages for 3 days, given ad libitum access to food, and evaluated for ambulatory activity using an OPTO-M3 sensor system (Columbus Instruments). A score of ambulatory activity was determined by consecutive photobeam breaks occurring in adjacent beams that were counted for 60 consecutive seconds for each of the twelve cages, cycling five times per hour during two complete light/dark cycles. Indirect calorimetry was measured in the same mice using an opencircuit Oxymax system (Columbus Instruments). Mice were individually housed in Plexiglass cages through which air of a known $\mathrm{O}_{2}$ concentration was passed at a constant flow rate. After a 48-hour acclimation period, the exhaust air in each cage was consecutively sampled for 60 seconds every 12 minutes over a 72-hour period for mice in the fed state to determine $\mathrm{O}_{2}$ and $\mathrm{CO}_{2}$ concentrations. Body composition of 14-week-old male, ad libitum HFD-fed control and LIRKO mice was determined using DXA scanning (Lunar PIXImus2 Densitometer; GE Healthcare) following anesthesia with Avertin (tribromoethanol:tert-amyl alcohol, $0.015 \mathrm{ml} / \mathrm{g}$ i.p.; Sigma-Aldrich).

Blood and plasma analysis. Fed-state glucose levels were measured between 9:00 am and 11:00 am in tail vein blood samples (Ascensia Elite). Serum was collected in the morning from mice in a random fed state, unless specified otherwise. Insulin was measured using a rat insulin ELISA with mouse standards (Crystal Chem Inc.). Cholesterol, triglycerides (Stanbio Laboratory), and FFAs (Wako) were measured using colorimetric and enzymatic methods, respectively. FGF21 was measured by a mouse-specific ELISA (BioVendor Inc.).

Pyruvate challenge, insulin tolerance test, and glucose uptake in vivo. The pyruvate challenge test was performed 1 week after insertion of the Alzet pumps. Animals were injected i.p. with $2 \mathrm{~g}$ pyruvate/ $\mathrm{kg}$ of body weight 4 hours after food removal, and glucose was measured at $0,15,30,60$, and 120 minutes. Glucose uptake and insulin tolerance tests were performed 2 weeks after insertion of the Alzet pumps. 2-deoxy-D-[1, 2-C $\left.{ }^{14}(\mathrm{~N})\right]$ glucose $(10 \mu \mathrm{Ci} / 30 \mathrm{~g}$ of body weight) was injected with or without insulin $(0.75 \mathrm{U} / \mathrm{kg})$ into the orbital vein of anesthetized animals (tribromoethanol:tert-amyl alcohol, $0.015 \mathrm{ml} / \mathrm{g}$ i.p.) starting 2 hours after food removal. Glucose was measured in tail vein samples at $0,2,5,15,30$, and 45 minutes after injection. Samples of liver, skeletal muscle, perigonadal adipose tissue, s.c. adipose tissue, and iBAT were taken at the end of the experiment and snap-frozen. Radioactivity was extracted and counted as previously described (52).

Analysis of gene expression by quantitative RT-PCR. Total RNA was isolated using the RNeasy Mini kit (QIAGEN). RNA $(1 \mu \mathrm{g})$ was used for reverse transcription using the High Capacity cDNA reverse transcription kit (Applied Biosystems). cDNA was diluted 1:20 and used for quantitative RT-PCR (qPCR) using gene-specific primers and TaqMan probes (Life Technologies). Fluorescence was monitored and analyzed using an ABI
Prism 7900 HT sequence detection system (Applied Biosystems). The relative expression for each condition was calculated using PP1A for liver and $36 \mathrm{~B} 4$ or TBP for adipose tissue as the endogenous control. Heatmaps were generated using GenePattern software (Broad Institute).

In vivo insulin signaling. On day 14 after pump insertion, animals were fasted for 2 hours, anesthetized with Avertin ( $0.015 \mathrm{ml} / \mathrm{g}$ i.p.), and injected via the inferior vena cava with $5 \mathrm{U}$ insulin (humulin). Five minutes after the insulin bolus, tissues were removed and frozen in liquid nitrogen. Immunoblot analysis of insulin signaling molecules was performed using tissue homogenates prepared with a tissue homogenization buffer containing $25 \mathrm{mM}$ Tris- $\mathrm{HCl}$ ( $\mathrm{pH}$ 7.4), $10 \mathrm{mM} \mathrm{Na}$ VO 4, $100 \mathrm{mM} \mathrm{NaF}, 50$ mM Na4P2O7, 10 mM EGTA, 10 mM EDTA, 2 mM phenylmethylsulfonyl fluoride, and $1 \%$ nonidet-P40 supplemented with a protease inhibitor cocktail (Sigma-Aldrich). Protein expression data were quantified by densitometry using ImageJ software (NIH). Rabbit polyclonal anti-IRs were purchased from Santa Cruz Biotechnology Inc., and anti-AKT, antiphospho-AKT (Ser473), anti-phospho-ERK, and anti-phospho-IR antibodies were purchased from Cell Signaling Technology.

Histology analysis. Adipose tissues were fixed in $10 \%$ formalin buffer, and sections were stained with $\mathrm{H} \& \mathrm{E}$.

Triglyceride content. Lipids were extracted from the liver with a solution containing $\mathrm{KOH}$ and ethanol, and glycerol was determined by enzymatic measurement using the triglyceride quantification kit from Abnova, according to the manufacturer's instructions.

Calculations and statistics. All data are expressed as the mean \pm SEM. Statistical analysis between multiple groups was determined with 2-way or 3-way ANOVA using SigmaPlot 12.5 (Systat Software). When the data did not demonstrate equal variance, nonparametric statistical assessment was used, which consisted of (a) a pooled pairwise test on each variable, and (b) a Kruskal-Wallis test on the different groups, followed by pairwise Wilcoxon tests. Statistical comparisons between two independent groups were calculated by unpaired Student's $t$ tests. Statistical significance was defined at $P<0.05$.

\section{Acknowledgments}

This work was supported by NIH grants R01-DK-033201 and R01DK031036 and by a grant from Eli Lilly and Company.

Received for publication March 7, 2013, and accepted in revised form October 24, 2013.

Address correspondence to: C. Ronald Kahn, Section on Integrative Physiology and Metabolism, Joslin Diabetes Center, One Joslin Place, Boston, Massachusetts 02215, USA. Phone: 617.309.2635; Fax: 617.309.2487; E-mail: c.ronald.kahn@joslin.harvard.edu.

Brice Emanuelli's and Sara G. Vienberg's present address is: Novo Nordisk Foundation Center for Basic Metabolic Research, Copenhagen University, Copenhagen, Denmark.
1. Haslam DW, James WP. Obesity. Lancet. 2005; 366(9492):1197-1209.

2. Danaei G, et al. National, regional, and global trends in fasting plasma glucose and diabetes prevalence since 1980: systematic analysis of health examination surveys and epidemiological studies with 370 country-years and 2.7 million participants. Lancet. 2011;378(9785):31-40.

3. Reaven G, Abbasi F, McLaughlin T. Obesity, insulin resistance, and cardiovascular disease. Recent Prog Horm Res. 2004;59:207-223.

4. Haas JT, Biddinger SB. Dissecting the role of insulin resistance in the metabolic syndrome. Curr Opin Lipidol. 2009;20(3):206-210.
5. Inzucchi SE, et al. Management of hyperglycaemia in type 2 diabetes: a patient-centered approach. Position statement of the American Diabetes Association (ADA) and the European Association for the Study of Diabetes (EASD). Diabetologia. 2012; 55(6):1577-1596

6. Kharitonenkov A, Larsen P. FGF21 reloaded: challenges of a rapidly growing field. Trends Endocrinol Metab. 2011;22(3):81-86.

7. Kharitonenkov A, et al. FGF-21 as a novel metabolic regulator. J Clin Invest. 2005;115(6):1627-1635.

8. Badman MK, Koester A, Flier JS, Kharitonenkov A, Maratos-Flier E. Fibroblast growth factor 21-deficient mice demonstrate impaired adaptation to ketosis. Endocrinology. 2009;150(11):4931-4940. 9. Badman MK, Pissios P, Kennedy AR, Koukos G, Flier JS, Maratos-Flier E. Hepatic fibroblast growth factor 21 is regulated by PPARalpha and is a key mediator of hepatic lipid metabolism in ketotic states. Cell Metab. 2007;5(6):426-437.

10. Inagaki $\mathrm{T}$, et al. Endocrine regulation of the fasting response by PPAR $\alpha$-mediated induction of fibroblast growth factor 21. Cell Metab. 2007;5(6):415-425.

11. Galman C, et al. The circulating metabolic regulator FGF21 is induced by prolonged fasting and PPAR $\alpha$ activation in man. Cell Metab. 2008;8(2):169-174.

12. Lundasen $T$, et al. PPARalpha is a key regulator 
of hepatic FGF21. Biochem Biophys Res Commun. 2007;360(2):437-440

13. Hondares E, Rosell M, Gonzalez FJ, Giralt M, Iglesias R, Villarroya F. Hepatic FGF21 expression is induced at birth via PPAR $\alpha$ in response to milk intake and contributes to thermogenic activation of neonatal brown fat. Cell Metab. 2010; 11(3):206-212

14. Mai K, et al. Free fatty acids link metabolism and regulation of the insulin-sensitizing fibroblast growth factor-21. Diabetes. 2009;58(7):1532-1538.

15. Mraz M, et al. Serum concentrations and tissue expression of a novel endocrine regulator fibroblast growth factor- 21 in patients with type 2 diabetes and obesity. Clin Endocrinol (Oxf). 2009; 71(3):369-375.

16. Arner P, Pettersson A, Mitchell PJ, Dunbar JD, Kharitonenkov A, Ryden M. FGF21 attenuates lipolysis in human adipocytes - a possible link to improved insulin sensitivity. Growth Regul. 2008; 582(12):1725-1730.

17. Xu J, et al. Fibroblast growth factor 21 reverses hepatic steatosis, increases energy expenditure, and improves insulin sensitivity in diet-induced obese mice. Diabetes. 2009;58(1):250-259.

18. Adams AC, et al. The breadth of FGF21's metabolic actions are governed by FGFR 1 in adipose tissue. Mol Metab. 2013;2(1):31-37.

19 . Ding $X$, et al. $\beta$ Klotho is required for fibroblast growth factor 21 effects on growth and metabolism. Cell Metab. 2012;16(3):387-393.

20. Adams AC, Cheng CC, Coskun T, Kharitonenkov A. FGF21 requires $\beta$ klotho to act in vivo. PLoS One. 2012;7(11):e49977.

21. Kharitonenkov A, et al. FGF-21/FGF-21 receptor interaction and activation is determined by betaKlotho. J Cell Physiol. 2008;215(1):1-7.

22. Ogawa $Y$, et al. $\beta$ Klotho is required for metabolic activity of fibroblast growth factor 21. Proc Natl Acad Sci U S A. 2007;104(18):7432-7437.

23. Suzuki M, et al. $\beta$ Klotho is required for fibroblast growth factor (FGF) 21 signaling through FGF receptor (FGFR) 1c and FGFR3c. Mol Endocrinol. 2008;22(4):1006-1014.

24. Michael MD, et al. Loss of insulin signaling in hepatocytes leads to severe insulin resistance and progressive hepatic dysfunction. Mol Cell. 2000; 6(1):87-97.

25. Biddinger $\mathrm{SB}$, et al. Hepatic insulin resistance directly promotes formation of cholesterol gallstones. Nat Med. 2008;14(7):778-782.

26. Coskun T, et al. Fibroblast growth factor 21 corrects obesity in mice. Endocrinology. 2008; 149(12):6018-6027.

27. Berglund ED, et al. Fibroblast growth factor 21 controls glycemia via regulation of hepatic glucose flux and insulin sensitivity. Endocrinology. 2009; 150(9):4084-4093.

28. Fisher SJ, Kahn CR. Insulin signaling is required for insulin's direct and indirect action on hepatic glucose production. J Clin Invest. 2003;111(4):463-468.

29. Cannon B, Nedergaard J. Brown adipose tissue: function and physiological significance. Physiol Rev. 2004;84(1):277-359.

30. Veniant MM, et al. FGF21 promotes metabolic homeostasis via white adipose and leptin in mice. PLoS One. 2012;7(7):e40164.

31. Cohen SE, Tseng YH, Michael MD, Kahn CR. Effects of insulin-sensitising agents in mice with hepatic insulin resistance. Diabetologia. 2004; 47(3):407-411.

32. Wente W, et al. Fibroblast growth factor- 21 improves pancreatic $\beta$-cell function and survival by activation of extracellular signal-regulated kinase $1 / 2$ and Akt signaling pathways. Diabetes. 2006; 55(9):2470-2478

33. Fisher FM, et al. FGF2 1 regulates PGC-1 $\alpha$ and browning of white adipose tissues in adaptive thermogenesis. Genes Dev. 2012;26(3):271-281.

34. Cypess $\mathrm{AM}$, et al. Identification and importance of brown adipose tissue in adult humans. NEngl J Med. 2009;360(15):1509-1517.

35. Marken Lichtenbelt WD, et al. Cold-activated brown adipose tissue in healthy men. N Engl J Med. 2009; 360(15):1500-1508.

36. Virtanen KA, et al. Functional brown adipose tissue in healthy adults. $N$ Engl J Med. 2009; 360(15):1518-1525.

37. Tseng YH, Cypess AM, Kahn CR. Cellular bioenergetics as a target for obesity therapy. Nat Rev Drug Discov. 2010;9(6):465-482.

38. Semple RK, Chatterjee VK, O'Rahilly S. PPAR $\gamma$ and human metabolic disease. J Clin Invest. 2006; 116(3):581-589.

39. Makimattila S, Nikkila K, Yki-Jarvinen H. Causes of weight gain during insulin therapy with and without metformin in patients with Type II diabetes mellitus. Diabetologia. 1999;42(4):406-412.
40. Dutchak PA, et al. Fibroblast growth factor-21 regulates PPAR $\gamma$ activity and the antidiabetic actions of thiazolidinediones. Cell. 2012;148(3):556-567.

41. Cohen $\mathrm{P}$, et al. Role for stearoyl-CoA desaturase-1 in leptin-mediated weight loss. Science. 2002; 297(5579):240-243.

42. Biddinger SB, Almind K, Miyazaki M, Kokkotou E, Ntambi JM, Kahn CR. Effects of diet and genetic background on sterol regulatory element-binding protein-1c, stearoyl-CoA desaturase 1 , and the development of the metabolic syndrome. Diabetes. 2005;54(5):1314-1323.

43. Ntambi JM, et al. Loss of stearoyl-CoA desaturase-1 function protects mice against adiposity. Proc Natl Acad Sci U S A. 2002;99(17):11482-11486.

44. Ntambia JM, Miyazaki M. Regulation of stearoylCoA desaturases and role in metabolism. Prog Lipid Res. 2004;43(2):91-104.

45. Biddinger SB, Miyazaki M, Boucher J, Ntambi JM, Kahn CR. Leptin suppresses stearoyl-CoA desaturase 1 by mechanisms independent of insulin and sterol regulating element-binding protein-1c. Diabetes. 2006;55(7):2032-2041.

46. Yoon JC, et al. Control of hepatic gluconeogenesis through the transcriptional coactivator PGC-1. Nature. 2001;413(6852):131-138.

47. Foretz M, Guichard C, Ferré P, Foufelle F. Sterol regulating element binding protein-1c is a major mediator of insulin action on the hepatic expression of glucokinase and lipogenesis-related genes. Proc Natl Acad Sci U S A. 1999;96(22):12737-12742.

48. Yabe D, Komuro R, Liang G, Goldstein JL, Brown MS. Liver-specific mRNA for Insig-2 down-regulated by insulin: implications for fatty acid synthesis. Proc Natl Acad Sci U S A. 2003;100(6):3155-3160.

49. Pothoff MJ, et al. FGF21 induces PGC-1 alpha and regulates carbohydrate and fatty acid metabolism during the adaptive starvation response. Proc Natl Acad Sci U S A. 2009;106(26):10853-10858.

50. Zhang Y, et al. The link between fibroblast growth factor 21 and sterol regulatory element binding protein 1c during lipogenesis in hepatocytes. $\mathrm{Mol}$ Cell Endocrinol. 2011;342(1-2):41-47.

51. Fisher FM, et al. Integrated regulation of hepatic metabolism by fibroblast growth factor 21 (FGF21) in vivo. Endocrinology. 2011;152(8):2996-3004.

52. An D, et al. TBC1D1 regulates insulin- and contraction-induced glucose transport in mouse skeletal muscle. Diabetes. 2010;59(6):1358-1365. 